\title{
The future look at the Galaxy with the Galactic Explorer with a Coded Aperture Mask Compton Telescope (GECCO)
}

\section{Elena Orlando, for the GECCO Collaboration}

Department of Physics, University of Trieste and National Institute of Nuclear Physics via Valerio 2, 34127 Trieste, Italy

Kavli Institute for Particle Astrophysics and Cosmology and Hansen Experimental Physics Laboratory, Stanford University

Lomita Mall 452, Stanford, CA, U.S.A.

E-mail: orlandele@gmail.com

In the past 15 years, observations of the Galaxy at high energies by Fermi-LAT, AGILE, INTEGRAL and very recently by NuSTAR and eROSITA have been shown to be very exciting, allowing discoveries of a variety of objects and unexpected breakthroughs. However, from a few hundreds of $\mathrm{KeV}$ to several tens of $\mathrm{MeV}$, the Galaxy remains poorly explored. In this energy range the lack of sufficiently sensitive instruments limits potential discoveries and challenges our understanding of the Galactic high-energy processes and sources. To solve this issue, GECCO is a new mission concept that will allow high-sensitivity observations of the sky from $\sim 50 \mathrm{KeV}$ to $\sim 10 \mathrm{MeV}$. It combines a coded aperture mask technique that provides high angular resolution for source detection, and a Compton telescope that provides high-sensitivity measurements of diffuse emissions. Such a combination enables efficient separation between sources and diffuse emissions. A GECCO-like mission has the potential of answering open questions and leading to new discoveries. Among the most recent challenges regarding the Galaxy, sensitive observations at $\mathrm{MeV}$ energies with unprecedented high resolution will open a new window in understanding complicated regions such as the inner Galaxy, the origin of the Fermi Bubbles, the origin of the $511 \mathrm{keV}$ line, and it will provide new insights on element formation in dynamical environments, on possible Galactic winds, and on the mechanisms of propagation of the low-energetic cosmic rays, their sources and their role on the Galaxy evolution. 


\section{Introduction}

In the past 15 years, observations of the Galaxy in x-rays and gamma rays have allowed exciting discoveries of a variety of objects and also unexpected breakthroughs. However, from a few hundreds of $\mathrm{keV}$ to several tens of $\mathrm{MeV}$, the Galaxy remains poorly explored due to the lack of sufficiently sensitive instruments in this energy range. Moreover, past instruments in the $\mathrm{MeV}$ energy range did not have enough angular resolution to disentangling sources from diffuse emission.

We present a new mission concept for a gamma-ray telescope in the $\mathrm{MeV}$ energy range, called GECCO.

\section{The GECCO Concept}

GECCO is a fully innovative mission concept in the energy band from $50 \mathrm{keV}$ to $10 \mathrm{MeV}$ [1]. It combines a coded mask telescope that provides high angular resolution for source detection, and a Compton telescope that provides high-sensitivity measurements of diffuse emissions and a larger field of view.

Such a combination enables efficient separation between sources and diffuse emissions, and among sources in crowded regions.

\section{GECCO Objectives}

The objectives for Galactic science are the following:

a) Allowing a deep look at the Galactic center with the aim of understanding the nature of the central super-massive black hole and the Fermi LAT Galactic Center excess (e.g. [2, 3]).

b) Detecting known and new sources thanks to the capability of disentangling and resolving sources and of distinguishing sources from the truly diffuse emission.

c) Investigating the origin of the gamma-ray Fermi Bubbles (e.g. [4]) and the x-ray Bubbles (e.g. [5]).

d) Tracing low-energetic cosmic rays, their propagation, their sources, and their role in the Galaxy evolution and star formation by detecting both continuum emission and de-excitation nuclear lines (e.g. [6] and references therein).

e) Allowing the study of the Galactic chemical evolution and the sites of nucleosynthesis of elements (e.g. [7]), and clarifying the origin of the $511 \mathrm{keV}$ electron-positron annihilation line (e.g. [8]).

\section{Conclusions}

A GECCO-like mission has the capability of answering open questions that other missions and multifrequency observations do not have, and it has the potential of leading to new discoveries.

\section{References}

1. A. Moiseev for the GECCO Collaboration, these proceedings

2. Hooper, Dan and Goodenough, Lisa, Physics Letters B 697 (2011) 412

3. A. Coogan, A. Moiseev, L. Morrison and S. Profumo, arXiv:2101.10370 (2021)

4. Su, M., \& Finkbeiner, D. P. (2012), The Astrophys-ical Journal, Vol. 753, 61

5. Orlando, Grenier, Tatischeff et al. Astro2020: Decadal Survey on Astronomy and Astrophysics, science white papers, no. 151; BAAS Vol. 51, Issue 3, id. 151 (2019)

6. Predehl, P., et al. Nature 588, 227-231 (2020) 
7. Tatischeff, V. and Gabici, S. (2018) Annual Review of Nuclear and Particle Science, vol. 68 , issue 1 , pp. $377-404$

8. C. Haymes, G.D., Walraven, C.A., Meegan, R.D., Hall, F.T. Djuth and D.H. Shelton, Astrophysical Journal201 (1975) 593

9. G. Weidenspointner et al., Nature 451(2008) 159 